\title{
German tariffs for the ICECAP-Supportive Care Measure (ICECAP-SCM) for use in economic evaluations at the end of life
}

\author{
Judith Dams ${ }^{1}$ - Elisabeth Huynh ${ }^{2}$. Steffi Riedel-Heller ${ }^{3} \cdot$ Margrit Löbner $^{3} \cdot$ Christian Brettschneider $^{1}$. \\ Hans-Helmut König ${ }^{1}$
}

Received: 23 April 2020 / Accepted: 18 December 2020 / Published online: 21 January 2021

(c) The Author(s) 2021

\begin{abstract}
Objectives Economic evaluations often use preference-based value sets (tariffs) for health-related quality of life to quantify health effects. For wellbeing at the end of life, issues beyond health-related quality of life may be important. Therefore, the ICECAP Supportive Care Measure (ICECAP-SCM), based on the capability approach, was developed. A validated German ICECAP-SCM version was published recently. However, tariffs for the German ICECAP-SCM are not available. Therefore, the aim was to determine tariffs for the ICECAP-SCM based on preferences of the German general population.

Methods An online sample of 2996 participants completed a best-worst scaling (BWS) and a discrete choice experiment (DCE). BWSs required participants to choose the best and worst statement within the same capability state, whereas DCEs required participants to trade-off between two capability states. First, BWS and DCE data were analyzed separately. Subsequently, combined data were analyzed using scale-adjusted conditional logit latent class models. Models were selected based on the stability of solutions and the Bayesian information criterion.

Results The two latent class model was identified to be optimal for the BWS, DCE, and combined data, and was used to derive tariffs for the ICECAP-SCM capability states. BWS data captured differences in ICECAP-SCM scale levels, whereas DCE data additionally explained interactions between the seven ICECAP-SCM attributes.

Discussion The German ICECAP-SCM tariffs can be used in addition to health-related quality of life to quantify effectiveness in economic evaluations. The tariffs based on BWS data were similar for Germany and the UK, whereas the tariffs based on combined data varied. We would recommend to use tariffs based on combined data in German evaluations. However, only results on BWS data are comparable between Germany and the UK, so that tariffs based on BWS data should be used when comparing results between Germany and the UK.
\end{abstract}

Keywords Value set $\cdot$ Capability $\cdot$ ICECAP-SCM $\cdot$ End-of-life $\cdot$ Best-worst scaling $\cdot$ Discrete choice experiment

Supplementary Information The online version contains supplementary material available at https://doi.org/10.1007/s1019 8-020-01260-2.

Judith Dams

j.dams@uke.de

1 Department of Health Economics and Health Services Research, Hamburg Center for Health Economics, University Medical Center Hamburg-Eppendorf, Martinistr. 52, 20246 Hamburg, Germany

2 Department of Health Service Research and Policy, Research School of Population Health, Australian National University, Canberra, Australia

3 Institute of Social Medicine, Occupational Health and Public Health (ISAP), University of Leipzig, Leipzig, Germany

\section{Introduction}

According to current guidelines for clinical trials and economic evaluations, patient-reported outcomes should be evaluated in addition to clinical outcomes [1]. Most patient-reported outcomes focus on health-related quality of life. However, studies focusing on health outcomes like health-related quality of life neglect other relevant issues of wellbeing, such as personal wishes or needs [2, 3]. In particular, the exclusive focus on aspect of healthrelated quality of life becomes less relevant for informing decision-making at the end of life. Instead, more care is needed and persons often suffer physically and have individual needs, such as financial issues, as well as wishes for the funeral or wellbeing of family and friends [4-6]. 
Therefore, measures of health-related quality of life may be less suitable to assess effects of interventions at the end of life [7]. Consequently, other concepts are important to measure effects in economic evaluations of interventions at the end of life, especially for groups of people whose needs are insufficiently reflected by the concept of healthrelated quality of life.

In contrast to the concept of health-related quality of life, the capability approach offers the possibility to focuses on the capability of persons to achieve wellbeing instead of utility [8-10]. Thus, the core focus is on what persons are able to do and who they are able to be, depending on what is important to them in life. Measures of wellbeing based on the capability approach are thus based on subjective perception, so that preferences of individual persons have to be determined. It can therefore be assumed that subjective preferences have a major impact on wellbeing.

A number of measures of capability have been published [11-13]. Some of these measures are suitable for generic use [11, 12], while other have been adapted to specific populations $[13,14]$. In principle, capability can be measured for all persons, thus the capability approach complements commonly used health-related quality of life measures, such as the EQ-5D [15, 16]. As the capability approach focuses on what persons are able to do and be [8-10], capability measures can be used to represent the quality of lifetime experienced at the end of life [17]. Therefore, the ICECAPSupportive Care Measure (ICECAP-SCM) has been developed recently for use in the evaluation of palliative and supportive care interventions [18]. The descriptive system of the ICECAP-SCM was developed using in-depth interviews with those at various points along the trajectory towards end of life. It consists of seven attributes relevant for capability at the end of life: choice (about my life and care), love and affection, physical suffering, emotional suffering, dignity, support, and preparation (making the preparations I want to make). The attributes are described by four ordinal levels "never (1)", "rarely (2)", "sometimes (3)" and "most of the time (4)" (levels for the ICECAP-SCM attributes 'physical suffering' and 'emotional suffering' are coded reverse). Overall, it is possible to describe $16,384\left(4^{7}\right)$ different capability states with " 1111111 " representing the no capability and "4444444" the full capability at the end of life [18].

The ICECAP-SCM can be used to measure the capability at the end of life of a single person. Furthermore, it is possible to compare the capabilities of different interventions with each other on the basis of tariffs. Thereby, the value of each of the capability states can be evaluated within a representative sample of the general population. To use the ICECAP-SCM in economic evaluation, tariffs are scaled between 0 and 1, representing no capability and full capability, respectively. Tariffs are available for the validated UK version of the ICECAP-SCM [19]. The tariffs were derived using a profile case best-worst-scaling (BWS) as well as a discrete choice experiment (DCE) valuation exercise.

In general, a BWS task comprises a single alternative or profile (e.g., end of life capability state) described by a combination of each attributes at a specific level. Participants are shown several such hypothetical scenarios or sets in which the profiles are varied by the attribute level scales and which are determined based on a statistical design. In DCEs, participants are presented with more than one profile in the same set, and are required to choose the most acceptable. Thus, DCEs enable participants to compare several profiles simultaneously [20] and BWS ask participants to choose the best and worst statement within the same profile [21]. DCE data analyzed under the random utility framework are therefore useful to derive compensatory trade-offs between several alternatives. However, for participants of a DCE, it may be difficult to understand the task [22]. For the valuation of quality of life outcomes, it has been suggested that profile case BWS is a more intuitive method for eliciting health state preferences, as it presents the respondent with an easierto-understand choice task than traditional DCEs [20,23]. In the case of the ICECAP-SCM in particular, the BWS has been shown to be feasible for use at end of life among patients and proxies using a think aloud study [24]. The respective advantages and disadvantages of BWS and DCE is an ongoing discussion [25]. When deriving tariffs for the UK version of the ICECAP-SCM, 6020 participants of a representative survey of the UK general population were asked to imagine their end of life [19]. For the BWS, the best and worst statement of a set of seven attributes of the ICECAP-SCM were chosen. For the DCE, participants were given a second set of seven attributes of the ICECAPSCM, which had to be compared with the previous set.

Recently, a German version of the ICECAP-SCM has been introduced and validated [26]. Yet, tariffs for the German version of the ICECAP-SCM have not yet been made available. However, it might not be appropriate to use the UK tariffs in Germany, due to possible cultural differences between the UK and other countries, which have been seen in studies comparing health-related quality of life tariffs. In a recently published research article comparing tariffs of the EQ-5D across different countries [27], the authors concluded that country-specific tariffs should be used to evaluate treatment effects. Indeed cultural differences in valuing health-related quality of life are well known, because culture defines the requirements and expectations for a meaningful life [28]. Likewise, culture may influence capability at the end of life. Therefore, country-specific tariffs of the ICECAP-SCM are needed. Thus, the aim of the current study was to determine tariffs for the ICECAP-SCM based on representative data for the German general population. 


\section{Methods}

\section{Experimental design}

The experimental design of the BWS and DCE was the same as that used for the UK valuation of the ICECAPSCM, so that results could be compared $[19,29]$. There are $16,384\left(4^{7}\right)$ possible ICECAP-SCM capability end of life states. The number of scenarios was reduced without loss of information using a Bayesian D-efficient design, using priors from 100 respondents from the UK, and by minimizing the variance-covariance matrix of the maximum likelihood estimator. The final design consisted of 16 sets to be completed by each respondent with each set accompanied by both a BWS and DCE task. For the BWS task in each scenario or set, participants were asked to choose the most and least acceptable statements out of seven attributes of one ICECAP-SCM end of life state. Different scenarios presented profiles with varying attribute levels on an ordinal four level scale with levels "never (1)", "rarely (2)", "sometimes (3)" and "most of the time (4)". For the DCE, participants compared an additional end of life profile described by attributes at the middling state (level 2 and 3) with the previous profile from the BWS, and were asked to choose their preferred scenario. The design ensured that the first eight scenarios were the same for all participants in the study. The other eight scenarios were drawn from one of five blocks of eight sets that were randomly assigned to respondents. Blocks were built to reduce the number of sets per participant from $n=48$ to $n=16$. Confounding between individuals and blocks was prevented by avoiding correlations between different blocking variables and the design attributes. The design was developed in Ngene: further details are available in [19].

\section{Socio-demographic and end of life parameters}

Socio-demographic parameters in the analysis included age, gender, education, employment status, professional qualification and marital status. Furthermore, parameters to capture the specific situation at the end of life were evaluated. Religiosity was measured by a visual analogue scale between 0 and 10, representing extreme non-religiosity and extreme religiosity, respectively. Furthermore, participants were asked if someone close to them had died in the last 2 years, if they themselves were diagnosed with a life-limiting illness, or if they care/had cared for someone with a life-limiting illness.

\section{Sampling and piloting}

The survey was conducted using an online panel by the external market research institute, USUMA GmbH, Berlin, Germany. The sample of the online panel was drawn representatively with regard to age, gender and federal state from the adult German general population. Only participants with a statement of consent were approved for the survey and were asked to fill in the BWS and DCE questionnaire. Participants with a fill-in time of less than $3.5 \mathrm{~min}$ ( $13 \mathrm{~s}$ per set) were excluded from the analysis in order to ensure quality of the data.

The questionnaire of the survey was based on the validated German version of the ICECAP-SCM [26] and was pilot tested in order to assess the difficulty and the comprehensibility of the tasks for participants. Pilot testing was conducted with 13 employees of the Department of Health Economics and Health Services Research (University Medical Center Hamburg-Eppendorf, Hamburg, Germany), and a further 50 participants of the online panel were interviewed subsequent to their participation in the survey. Participants of the piloting test were asked to rate questions in terms of difficulty and intimacy. Participants were given the option to deny answers of life-limiting illness, death, and religiosity.

\section{Statistical analyses}

DCEs and BWSs are based on random-utility models [30], which aim to model the choices of individuals among discrete sets of alternatives, assuming that preferences among these alternatives can be described by a utility function [20]. Thus, preferences among alternatives of the ICECAP-SCM questionnaire can be described by a conditional logit model representing the particular relevance of each ICECAP-SCM attribute for capability [19, 31]. First, the BWS and DCE data were analyzed using conditional logit models. Following this, the similarity between BWS and DCE data was evaluated to assess whether it is possible to combine both sources of choice data. Again, conditional logit models were used to analyze combined data. In order to increase representativeness of the results for the German general adult population, the German Census 2011 [32] was used to generate weights that compensate for underrepresentation and overrepresentation of observations by means of age, gender, and education. All conditional logit models used were estimated using Latent Gold 5.1 with Choice and Advanced/Syntax addon. Data preparation and further analyses were conducted using R 3.5.1. 


\section{Analysis of BWS and DCE data}

BWS data were analyzed using a partial rank-ordered scale-adjusted conditional logit latent class model, where the best statement of each set was selected first, and the worst statement from those remaining. Sign changes were used to reflect the contrary meaning of the best and worst decisions. Scale differences between the best and worst decision were adjusted by including a scaling factor in the model. Attributes of the ICECAP-SCM were included as independent variables to evaluate their influence on capability at the end of life. Respondents with similar response patterns were classified using latent classes. Models with convergence towards a stable solution (identifiable and same model fit across different starting values and seeds), low Bayesian Information Criterion (BIC) and judged to provide meaningful classes, were selected. Covariates, such as socio-demographic characteristics and variables concerning statements for the end of life, were first included in the model independently as single parameters. In a second step, multiple socio-demographic parameters were tested on their influence on capability. Variables were integrated using a forward algorithm (Wald test significance level of 5\%).

DCE data were analyzed using scale-adjusted conditional logit latent class models. Again, attributes of the ICECAPSCM were included as independent variables to determine their influence on capability at the end of life. Models with convergence towards a stable solution and the lowest BIC were selected. Again, socio-demographic characteristics and variables concerning statements for the end of life were included as single parameters first, and then as multiple parameters using a forward algorithm.

\section{Analysis of combined data}

BWS and DCE data can be combined if both choice data are shown to have the same data generating process. If the number of classes and the class-specific influence of attributes of the ICECAP-SCM on capability is similar, it may be possible to combine the two sources of choice data. Model coefficients of the separate analyses of BWS and DCE data represented the influence of scale levels of the ICECAPSCM attributes on capability. Therefore, coefficients based on BWS data were compared with coefficients based on DCE data using Pearson correlations and scatterplots. The consistency of results between BWS and DCE data was assumed for a linear relationship between coefficients of BWS and DCE data, which indicated a similar influence of scale levels of attributes on capability.

The combined data was first analyzed using a scaleadjusted conditional logit latent class model with main effects. Two-way interactions used to derive tariffs for the ICECAP-SCM in the UK were not included, because solutions were not robust. Thus, the capability utility function $U_{n}$ for a respondent $n$ is represented by the following equation:

$U_{n}=\exp \left(y_{\mathrm{BWS}}+y_{\mathrm{BWS}-\mathrm{DCE}}\right)\left[\mathrm{Const}+\sum_{i} \mathrm{ASC}_{i}+\sum_{i} \beta_{i} X_{\mathrm{in}}\right]$

where Const represents the effects coded intercept for the DCE data and $\mathrm{ASC}_{i}$ represents the effect coded intercept of the attribute $i$ of the ICECAP-SCM. $X_{i}$ are indicators for attribute $i$ for $k=1, \ldots, 7$. Differences in scaling between DCE and BWS data were adjusted by the scaling factor $y_{\mathrm{BWS}-\mathrm{DCE}}$. Differences in scaling between best and worst data were captured by sign changes. Furthermore, an additional scaling factor $y_{\mathrm{BWS}}$ was used to adjust scale differences between best and worst data. Models with convergence towards a stable solution and the lowest BIC were selected. Again, socio-demographic characteristics and variables concerning statements for the end of life were included as single parameters first, and then as multiple parameters using a forward algorithm.

\section{Determination of tariffs}

First, for each of the $16,484\left(4^{7}\right)$ states, class-specific values for BWS, DCE and combined data were calculated, based on the results of the conditional logit latent class models. Then, the average values across the latent classes were calculated by taking the weighted mean of class-specific values across classes. Finally, the value for each state was transformed to a scale between 0 and 1 by subtracting the value for the no capability state " 1111111 " from the respective value, and by dividing this difference with the difference of the value for the full capability state " 4444444 " and the value for the no capability state " 1111111 ". Descriptive statistics and the intraclass correlation (ICC) were used to compare tariffs based on BWS, DCE and combined data.

\section{Ethical statement}

According to the ethics committee of the Hamburg Medical Chamber, an ethics approval was not required as only anonymized survey data was used.

\section{Results}

Of the 6249 persons contacted, 4329 (69\%) participants completed the questionnaire of the survey. Of those participants who completed the survey, 1159 (19\%) had a 
fill-in time of less than $3.5 \mathrm{~min}$ and/or did not complete a statement of consent, and were excluded from the analysis. On average, included participants had a fill-in time of $18 \mathrm{~min}$. As participants were surveyed representative for the population size of German federal states, participants living in already overrepresented federal states were no longer surveyed. Between reaching the representative population size of German federal states and closing the survey for particular federal states, another 101 (2\%) participants living in the particular federal states were interviewed, thus they were excluded from analysis. Of the remaining 3069 participants, 73 (1\%) had missing data and were also excluded from the analysis. Overall, 2996 (47\%) participants were analyzed.

Sample characteristics differed in age and education compared with the German general adult population. Persons younger than 35 years and persons older 55 years were underrepresented. Furthermore, survey respondents were more likely to have a higher education level (i.e., intermediate secondary school, technical college, A-level exam) compared with the German general adult population. Therefore, sample characteristics in age, gender and education were adjusted to data from the German Census 2011 [32] using population-specific weights. Mean age of the weighted sample was 2.5 years younger. Furthermore, in the weighted sample, a higher proportion had received a secondary school examination, and a lower proportion an A-level exam, compared with pre-weighting characteristics (Table 1).

\section{BWS}

BWS data were analyzed using a model with two latent classes. While the BIC continued to improve for the third and fourth class, the two latent class model has been kept simpler, with three or four latent classes providing similar content and non-meaningful differences beyond the two latent class model. The final BWS model controlled for differences in gender, age, education, professional qualification, employment, diagnosis of a life-limiting illness, and care for a person with a life-limiting illness in the class membership function. Coefficients for scale levels of attributes were similar in class 1 and differed more widely in class 2 (Table 2). For respondents in class 1, differences in coefficients for scale levels were highest for the attributes 'physical suffering' and 'dignity', whereas for respondents in class 2, differences in coefficients for scale levels were highest for the attribute 'love and affection'. Thus, for respondents in class 1 'physical suffering' and 'dignity' had the highest influence on capability at the end of life, whereas respondents in class 2 considered 'love and affection' to have the highest influence.

\section{DCE}

Solutions based on the DCE data were robust for scaleadjusted conditional logit models with two and three latent classes including age, education, and religiosity as covariates. The BIC was lowest for a model with three latent classes. However, the third class was similar in class composition of respondents and provided similar content. For respondents in class 1 , differences in coefficients for scale levels were highest for the attribute 'love and affection', whereas for respondents in class 2 differences in coefficients for scale levels were highest for the attribute 'dignity' (Table 3). Thus, for respondents in class 1 'love and affection' had the highest influence on capability at the end of life, whereas respondents in class 2 considered 'dignity' to have the highest influence.

\section{Combined model}

Scatterplots on coefficients for two, three and four latent classes revealed a linear relationship between coefficients based on BWS data and coefficients based on DCE data (Fig. 1). Furthermore, Pearson's correlation coefficients were $0.91,0.90$ and 0.82 for two, three and four latent classes, respectively.

Combined data was analyzed using a scale-adjusted conditional logit model with two latent classes. The final model for combined data controlled for differences in education, employment, diagnosis of a life-limiting illness, caring for someone with a life-limiting illness and religiosity as covariates (Table 4). For respondents in class 1, differences in coefficients for scale levels were highest for the attributes 'support' and 'dignity', whereas for respondents in class 2 differences in coefficients for scale levels of attributes were small. Thus, for respondents in class 1 'support' and 'dignity' had the highest influence on capability at the end of life, whereas for respondents in class 2, all attributes influenced capability similarly.

\section{Tariffs}

Results of the scale-adjusted conditional logit models based on BWS, DCE and combined data were transformed into tariffs. Tariffs for each capability state can be calculated based on tariff increases and reductions represented in Table 5 or taken from the Appendix Excel worksheet. For example, the tariff for the capability state " 1111111 " based on BWS data is calculated as follows: The first row of Table 5 provide tariff increases and reductions of $0.008,-0.004,-0.003$, $0.019,-0.040,-0.007$ and 0.027 for 'never being able to make decisions', 'never being with people who care about you', 'always experiencing physical suffering', 'always experiencing emotional suffering', 'never experiencing dignity', 
Table 1 Socio-demographic and end of life characteristics of the sample $(n=2996)$

\begin{tabular}{|c|c|c|}
\hline Characteristics & Sample & Weighted sample ${ }^{a}$ \\
\hline \multicolumn{3}{|l|}{ Gender $n(\%)$} \\
\hline Male & $1436(47.9)$ & $1471(49.1)$ \\
\hline Female & $1554(51.9)$ & $1519(50.7)$ \\
\hline Diverse & $6(0.2)$ & $6(0.2)$ \\
\hline Age mean (SD) & $52.3(12.1)$ & $49.7(12.1)$ \\
\hline \multicolumn{3}{|l|}{ Education $n(\%)$} \\
\hline Not graduated & $5(0.2)$ & $23(0.8)$ \\
\hline Secondary school & $443(14.8)$ & $871(29.1)$ \\
\hline Intermediate secondary school & $1237(41.3)$ & $1426(47.6)$ \\
\hline High school & 1307 (43.6) & $675(22.5)$ \\
\hline Other education & $4(0.1)$ & $1(0)$ \\
\hline \multicolumn{3}{|l|}{ Employment $n(\%)$} \\
\hline Employed & $1461(48.8)$ & $1574(52.6)$ \\
\hline Self-employed & $189(6.3)$ & $138(4.6)$ \\
\hline Housewife/househusband & $196(6.5)$ & $218(7.3)$ \\
\hline Not employed & $130(4.3)$ & $190(6.3)$ \\
\hline Retired & $856(28.6)$ & $721(24.1)$ \\
\hline Apprenticeship & $118(3.9)$ & $109(3.6)$ \\
\hline Other employment & $46(1.6)$ & $46(1.5)$ \\
\hline \multicolumn{3}{|l|}{ Professional qualification $n(\%)$} \\
\hline No professional qualification & $138(4.6)$ & $207(6.9)$ \\
\hline In professional qualification for less than 1 year & $23(0.8)$ & $39(1.3)$ \\
\hline In professional qualification for less than 2 years & $188(6.3)$ & $219(7.3)$ \\
\hline In professional qualification for less than 3 years & $1451(48.4)$ & $1782(59.5)$ \\
\hline Technical college & $359(12.0)$ & $305(10.2)$ \\
\hline University of applied science/polytechnic & $308(10.3)$ & $160(5.3)$ \\
\hline University & $461(15.4)$ & $236(7.9)$ \\
\hline Post-doc & $27(0.9)$ & $13(0.4)$ \\
\hline Other professional qualification & $41(1.3)$ & $35(1.2)$ \\
\hline \multicolumn{3}{|l|}{ Marital status $n(\%)$} \\
\hline Married/registered civil partner & $1551(51.8)$ & $1455(48.6)$ \\
\hline In relationship & $326(10.9)$ & $358(11.9)$ \\
\hline Single & $642(21.4)$ & $754(25.2)$ \\
\hline Divorced & $380(12.7)$ & $351(11.7)$ \\
\hline Widowed & $97(3.2)$ & $78(2.6)$ \\
\hline Religiosity mean (SD) & $3.6(5.7)$ & $3.52(5.1)$ \\
\hline \multicolumn{3}{|l|}{ Diagnosis of life limiting illness $n(\%)$} \\
\hline Yes & $105(3.5)$ & $109(3.6)$ \\
\hline No & $2815(94.0)$ & $2815(94.0)$ \\
\hline No answer & $76(2.5)$ & $72(2.4)$ \\
\hline \multicolumn{3}{|l|}{ Caring for someone with life limiting illness $n(\%)$} \\
\hline Yes & $115(3.8)$ & $108(3.6)$ \\
\hline No & $2842(94.9)$ & $2851(95.2)$ \\
\hline No answer & $39(1.3)$ & $37(1.2)$ \\
\hline \multicolumn{3}{|l|}{ Had someone close died in the last two years $n(\%)$} \\
\hline Yes & $1229(41.0)$ & $1171(39.1)$ \\
\hline No & $1735(57.9)$ & $1789(59.7)$ \\
\hline No answer & $32(1.1)$ & $37(1.2)$ \\
\hline
\end{tabular}

${ }^{\text {a }}$ The sample was adapted to the German general adult population by means of age, gender, and education using data from the German Census 2011 [32] 
Table 2 Results of scaleadjusted conditional logit model with two latent classes based on best-worst scaling data $(n=2996)$

\begin{tabular}{|c|c|c|}
\hline Parameter estimates & Class $1(26.7 \%)$ & Class $2(73.3 \%)$ \\
\hline \multicolumn{3}{|l|}{ Intercept ${ }^{* * *}$} \\
\hline Support & 0.388 & 0.239 \\
\hline Physical suffering & 0.989 & -0.148 \\
\hline Preparation & 0.214 & 0.442 \\
\hline Love and affection & 0.247 & -0.038 \\
\hline Choice & -0.227 & 0.274 \\
\hline Emotional suffering & -0.613 & 0.052 \\
\hline Dignity & -0.999 & -0.821 \\
\hline \multicolumn{3}{|l|}{ Choice*** } \\
\hline Never (1) & -0.243 & -1.850 \\
\hline Only a little (2) & -0.317 & -1.189 \\
\hline Some (3) & 0.101 & 0.605 \\
\hline Most (4) & 0.206 & 1.863 \\
\hline \multicolumn{3}{|l|}{ Love and affection $* * *$} \\
\hline Never (1) & -0.391 & -2.026 \\
\hline Only a little (2) & -0.264 & -0.713 \\
\hline Some (3) & 0.099 & 1.026 \\
\hline Most (4) & 0.224 & 2.604 \\
\hline \multicolumn{3}{|l|}{ Physical suffering $* * *$} \\
\hline Always (1) & 0.426 & -2.450 \\
\hline Often (2) & 0.631 & -1.702 \\
\hline Sometimes (3) & 0.080 & 0.116 \\
\hline Rarely (4) & -0.822 & 2.166 \\
\hline \multicolumn{3}{|l|}{ Emotional suffering $* * *$} \\
\hline Always (1) & 0.025 & -1.290 \\
\hline Often (2) & -0.531 & -1.231 \\
\hline Sometimes (3) & -0.321 & 0.342 \\
\hline Rarely (4) & -0.490 & 1.267 \\
\hline \multicolumn{3}{|l|}{ Dignity*** } \\
\hline Never (1) & -0.399 & -1.796 \\
\hline Only a little (2) & 0.108 & -0.543 \\
\hline Some (3) & 0.412 & 1.498 \\
\hline Most (4) & 0.869 & 2.968 \\
\hline \multicolumn{3}{|l|}{ Support*** } \\
\hline Never (1) & -0.530 & -2.367 \\
\hline Only a little (2) & -0.306 & -0.970 \\
\hline Some (3) & 0.202 & 0.869 \\
\hline Most (4) & 0.407 & 2.095 \\
\hline \multicolumn{3}{|l|}{ Preparation $* * *$} \\
\hline Not any (1) & -0.342 & -1.605 \\
\hline Only few (2) & 0.220 & -0.079 \\
\hline Some (3) & 0.456 & 0.795 \\
\hline Most (4) & 0.489 & 1.597 \\
\hline \multicolumn{3}{|l|}{ Gender*** } \\
\hline Male & 1.225 & -1.225 \\
\hline Female & 0.983 & -0.983 \\
\hline Diverse & -2.209 & 2.209 \\
\hline Age, years*** & -0.012 & 0.012 \\
\hline
\end{tabular}


Table 2 (continued)

\begin{tabular}{|c|c|c|}
\hline Parameter estimates & Class $1(26.7 \%)$ & Class $2(73.3 \%)$ \\
\hline \multicolumn{3}{|l|}{$\overline{\text { Education*** }}$} \\
\hline No education & 1.100 & -1.100 \\
\hline Secondary school & 0.099 & -0.099 \\
\hline Intermediate secondary school & -0.080 & 0.080 \\
\hline Technical college & -0.258 & 0.258 \\
\hline A level exam & -0.420 & 0.420 \\
\hline Other education & -0.441 & 0.441 \\
\hline \multicolumn{3}{|l|}{ Professional qualification* } \\
\hline No professional qualification & -0.043 & 0.043 \\
\hline In professional qualification for less than 1 year & 0.016 & -0.016 \\
\hline In professional qualification for less than 2 years & 0.246 & -0.246 \\
\hline In professional qualification for less than 3 years & -0.139 & 0.139 \\
\hline Technical college & -0.165 & 0.165 \\
\hline University of applied science/polytechnic & -0.026 & 0.026 \\
\hline University & -0.123 & 0.123 \\
\hline Post-doc & 0.266 & -0.266 \\
\hline Other professional qualification & -0.031 & 0.031 \\
\hline \multicolumn{3}{|l|}{ Employment** } \\
\hline Employed & -0.042 & 0.042 \\
\hline Self-employed & -0.119 & 0.119 \\
\hline Housewife/househusband & -0.169 & 0.169 \\
\hline Not employed & -0.019 & 0.011 \\
\hline Retired & 0.235 & -0.235 \\
\hline Apprenticeship & -0.200 & 0.200 \\
\hline Other employment & 0.376 & -0.376 \\
\hline \multicolumn{3}{|l|}{ Diagnosis of a life limiting illness $* * *$} \\
\hline Yes & 0.297 & -0.297 \\
\hline No & -0.231 & 0.231 \\
\hline No answer & -0.066 & 0.066 \\
\hline \multicolumn{3}{|l|}{ Caring for someone with life limiting illness $* * *$} \\
\hline Yes & 0.105 & -0.105 \\
\hline No & -0.290 & 0.290 \\
\hline No answer & 0.185 & 0.185 \\
\hline Class membership parameters & -0.379 & 0.379 \\
\hline Scaling factor BWS data*** & -1.113 & \\
\hline \multicolumn{3}{|l|}{ Goodness of fit } \\
\hline LL & $-155,322$ & \\
\hline Number of parameters & 95 & \\
\hline AIC & 310,834 & \\
\hline $\mathrm{BIC}$ & 311,405 & \\
\hline Number of choice sets & 95,872 & \\
\hline Number of subjects & 2996 & \\
\hline
\end{tabular}

All attributes and class membership covariates are effects coded. Reference levels reported here are calculated as the negative sum of other parameters of the same attribute. Be careful with the coding for 'physical suffering' and 'emotional suffering' (levels are coded reverse)

$B W S$ best-worst-scaling, $L L$ log-likelihood, AIC Akaike information criterion, BIC Bayesian information criterion.

$* p \leq 0.05, * * p \leq 0.01, * * * p \leq 0.001$ 
Table 3 Results of scale-adjusted conditional logit model with two latent classes based on discrete choice experiment data $(n=2996)$

\begin{tabular}{|c|c|c|}
\hline Parameter estimates & $\begin{array}{l}\text { Class 1 } \\
(66.8 \%)\end{array}$ & $\begin{array}{l}\text { Class } 2 \\
(33.2 \%)\end{array}$ \\
\hline \multicolumn{3}{|l|}{ Intercept } \\
\hline Set 1 & -0.156 & 0.156 \\
\hline Set 2 & 0.045 & -0.045 \\
\hline \multicolumn{3}{|l|}{ Choice*** } \\
\hline Never (1) & -0.732 & -0.919 \\
\hline Only a little (2) & -0.143 & 0.088 \\
\hline Some (3) & 0.274 & 0.225 \\
\hline Most (4) & 0.601 & 0.606 \\
\hline \multicolumn{3}{|l|}{ Love and affection $* * *$} \\
\hline Never (1) & -1.330 & -0.236 \\
\hline Only a little (2) & -0.105 & -0.290 \\
\hline Some (3) & 0.412 & 0.255 \\
\hline Most (4) & 1.024 & 0.271 \\
\hline \multicolumn{3}{|l|}{ Physical suffering*** } \\
\hline Always (1) & -0.756 & -0.349 \\
\hline Often (2) & -0.254 & -0.229 \\
\hline Sometimes (3) & 0.373 & 0.269 \\
\hline Rarely (4) & 0.638 & 0.309 \\
\hline \multicolumn{3}{|l|}{ Emotional suffering $* * *$} \\
\hline Always (1) & -0.395 & -0.313 \\
\hline Often (2) & -0.096 & -0.111 \\
\hline Sometimes (3) & 0.225 & 0.121 \\
\hline Rarely (4) & 0.265 & 0.303 \\
\hline \multicolumn{3}{|l|}{ Dignity*** } \\
\hline Never (1) & -0.805 & -2.763 \\
\hline Only a little (2) & -0.077 & -0.713 \\
\hline Some (3) & 0.235 & 1.033 \\
\hline Most (4) & 0.647 & 2.442 \\
\hline \multicolumn{3}{|l|}{ Support*** } \\
\hline Never (1) & -0.764 & -0.633 \\
\hline Only a little (2) & -0.087 & -0.270 \\
\hline Some (3) & -0.417 & -0.107 \\
\hline Most (4) & 0.433 & 1.010 \\
\hline \multicolumn{3}{|l|}{ Preparation $* * *$} \\
\hline Not any (1) & -0.528 & -0.409 \\
\hline Only few (2) & -0.050 & -0.084 \\
\hline Some (3) & 0.140 & 0.103 \\
\hline Most (4) & 0.438 & 0.390 \\
\hline Age, years*** & -0.196 & 0.196 \\
\hline \multicolumn{3}{|l|}{ Education $* * *$} \\
\hline No education & -0.876 & 0.876 \\
\hline Secondary schools & -0.095 & 0.095 \\
\hline Intermediate secondary school & 0.045 & -0.045 \\
\hline Advanced technical college certificate & -0.276 & 0.276 \\
\hline High school & -0.329 & 0.329 \\
\hline Other education & 1.530 & -1.530 \\
\hline
\end{tabular}

Table 3 (continued)

\begin{tabular}{|c|c|c|}
\hline Parameter estimates & $\begin{array}{l}\text { Class } 1 \\
(66.8 \%)\end{array}$ & $\begin{array}{l}\text { Class } 2 \\
(33.2 \%)\end{array}$ \\
\hline \multicolumn{3}{|l|}{ Religiosity*** } \\
\hline 0 (not religious) & -0.386 & 0.386 \\
\hline 1 & -0.429 & 0.429 \\
\hline 2 & -0.302 & 0.302 \\
\hline 3 & -0.207 & 0.207 \\
\hline 4 & -0.440 & 0.440 \\
\hline 5 & -0.304 & 0.304 \\
\hline 6 & -0.163 & 0.163 \\
\hline 7 & -0.394 & 0.394 \\
\hline 8 & -0.239 & 0.239 \\
\hline 9 & -0.377 & 0.377 \\
\hline 10 (very religious) & 0.080 & -0.080 \\
\hline No answer & 3.161 & -3.161 \\
\hline Class membership parameters*** & 1.753 & 1.753 \\
\hline \multicolumn{3}{|l|}{ Scaling factor } \\
\hline DCE data*** & -1.932 & \\
\hline \multicolumn{3}{|l|}{ Goodness of fit } \\
\hline LL & $-28,527$ & \\
\hline \#Parameters & 64 & \\
\hline AIC & 57,182 & \\
\hline $\mathrm{BIC}$ & 57,567 & \\
\hline Number of choice sets & 47,936 & \\
\hline Number of subjects & 2996 & \\
\hline
\end{tabular}

All attributes and class membership covariates are effects coded. Reference levels reported here are calculated as the negative sum of other parameters of the same attribute. Be careful with the coding for 'physical suffering' and 'emotional suffering' (levels are coded reverse)

$D C E$ discrete choice experiment, $L L$ log-likelihood, AIC Akaike information criterion, BIC Bayesian information criterion ${ }^{*} p \leq 0.05, * * p \leq 0.01, * * * p \leq 0.001$

'never being supported', and 'not being prepared', respectively. Thus, the tariff for the capability state "1111111" based on BWS data is calculated by the sum of the respective tariff increases and reductions: $0.008-0.004-0.003+0.0$ $19-0.040-0.007+0.027=0$. Tariffs for other capability states are derived by adapting the respective tariff increases and reductions. Thus, for example, the tariff for the capability state " 1121111 " is derived by substituting the tariff reduction of -0.003 for 'always experiencing physical suffering' by the tariff increase of 0.025 for 'often experiencing physical suffering'. Thus, the tariff for the capability state " $1121111 "$ is $0.008-0.004+0.025+0.019-0.040-0.00$ $7+0.027=0.028$ based on BWS data.

The tariffs were scaled between 0 and 1, representing the values for the no and full capability states "1111111" 


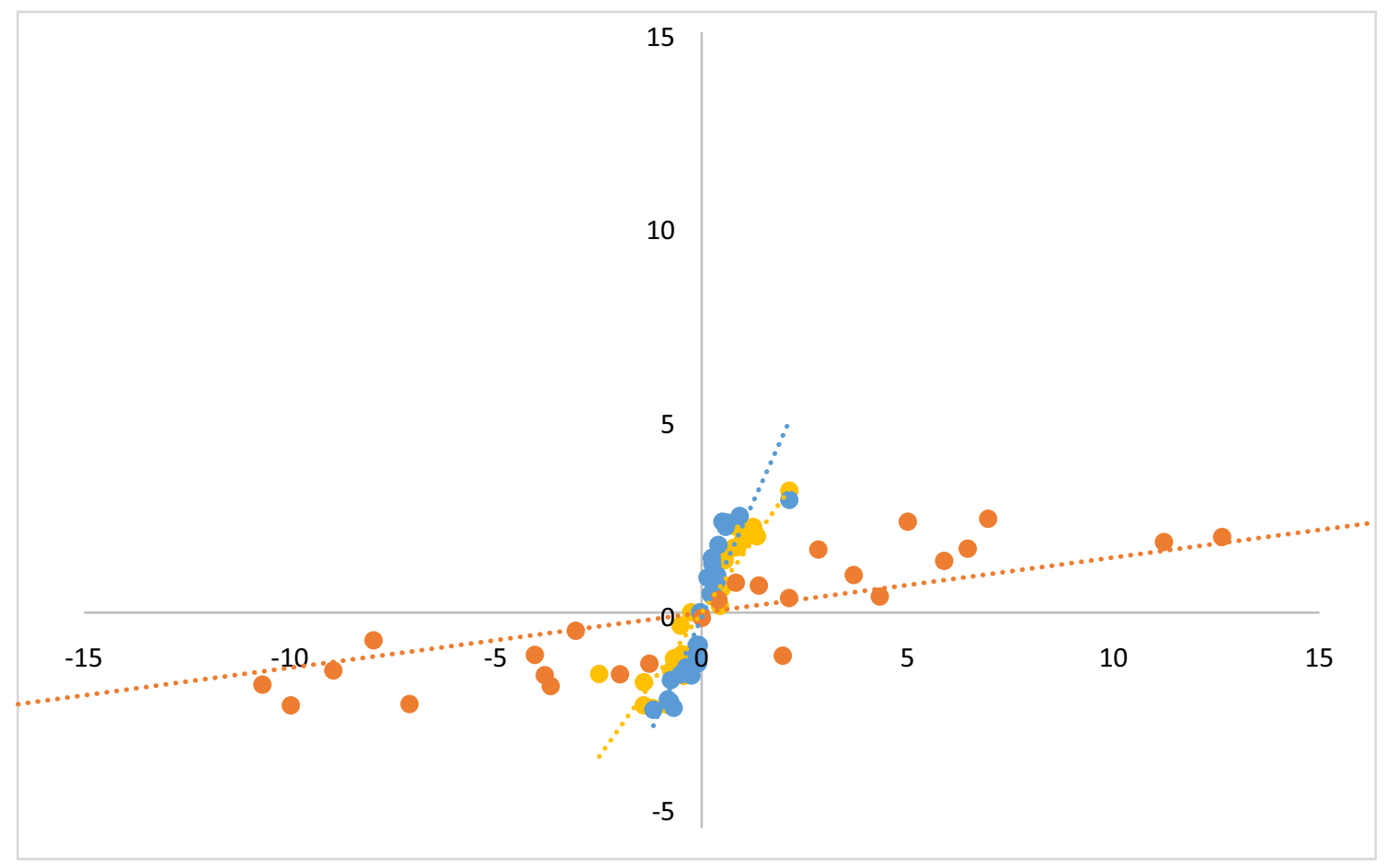

Fig. 1 Scatterplot of coefficients for scale levels of the ICECAP-SCM attributes of models based on DCE and BWS data. Comparison of coefficient for scale levels of scale-adjusted conditional logit models with two (yellow), three (blue) and four (orange) latent classes based on DCE ( $X$-axis) and BWS ( $Y$-axis) data. As coefficients represent the influence of scale levels of attributes on capability, a linear trend (dotted lines) indicate a similar influence of the scale levels for BWS and DCE data on capability

\section{Discussion}

the capability state "1112111" based on BWS data and combined data were negative, because respondents rated 'always experiencing emotional suffering' with smaller tariff increases of 0.019 and 0.013 than 'often experiencing emotional suffering' with tariff increases of 0.014 and - 0.006, respectively. As differences in tariff increases for both scale levels of the attribute 'emotional suffering' were small, social-cultural and linguistic differences were assumed to be responsible for these discrepancies. Participants never compared both scale levels of the attribute 'emotional suffering' directly with each other. Furthermore, in German the difference between 'always experiencing emotional suffering' and 'often experiencing emotional suffering' may not be pronounced as in English. Therefore, it was assumed that the tariff of the capability state " 1112111 " was very similar to the tariff of the capability state "1111111", and was thus replaced by zero.

Based on BWS, DCE and combined data, tariffs of all capability states were normally distributed with means and standard derivations (SD) of 0.48 (SD 0.15), 0.53 (SD 0.15) and 0.49 (SD 0.16), respectively. Furthermore, a significant intra-class correlation (ICC) between the tariffs of 0.894 was observed.
This article presents tariffs for the German version of the ICECAP-SCM in order to enable the use of this capability measure in economic evaluations. As the ICECAP-SCM is the first questionnaire to measure capability of persons at their end of life, it enables the evaluation of interventions and services using domains relevant for persons at the end of life.

The current study provides tariffs based on BWS, DCE and combined data. As methods were similar to those used for the UK valuation, the current study is the second study based on combined BWS and DCE data. Differences between tariffs based on BWS, DCE and combined data were small. All three tariffs were distributed normally with a similar mean. Furthermore, the ICCs between tariffs for BWS, DCE and combined data confirmed a strong correlation. However, the coefficients of the various scale levels of the ICECAP-SCM attribute to calculate tariffs varied, especially for those of the attribute 'dignity', which may be explained by the different focuses of the BWS and DCE. For BWS, single profiles are compared with each other, whereas sets of profiles are compared by DCEs. Both methods have advantages and disadvantages. Thereby, DCEs are 
Table 4 Results of scaleadjusted conditional logit model with two latent classes based on combined best-worst scaling and discrete choice experiment data $(n=2996)$

\begin{tabular}{|c|c|c|}
\hline Parameter estimates & Class $1(57.4 \%)$ & Class $2(42.6 \%)$ \\
\hline \multicolumn{3}{|l|}{ Intercept $* * *$} \\
\hline Support & -18.413 & -17.539 \\
\hline Physical suffering & -18.495 & -17.562 \\
\hline Preparation & -18.363 & -17.513 \\
\hline Love and affection & -18.451 & -17.531 \\
\hline Choice & -18.400 & -17.534 \\
\hline Emotional suffering & -18.432 & -17.578 \\
\hline Dignity & -18.449 & -17.546 \\
\hline DCE1 & 64.423 & 61.389 \\
\hline DCE2 & 64.580 & 61.415 \\
\hline \multicolumn{3}{|l|}{ Choice*** } \\
\hline Never (1) & -0.141 & -0.014 \\
\hline Only a little (2) & -0.084 & -0.014 \\
\hline Some (3) & 0.074 & 0.015 \\
\hline Most (4) & 0.152 & 0.013 \\
\hline \multicolumn{3}{|l|}{ Love and affection $* * *$} \\
\hline Never (1) & -0.128 & -0.006 \\
\hline Only a little (2) & -0.048 & -0.003 \\
\hline Some (3) & 0.000 & -0.009 \\
\hline Most (4) & 0.177 & 0.018 \\
\hline \multicolumn{3}{|l|}{ Physical suffering*** } \\
\hline Always (1) & -0.114 & 0.000 \\
\hline Often (2) & -0.067 & -0.004 \\
\hline Sometimes (3) & 0.034 & 0.005 \\
\hline Rarely (4) & 0.146 & -0.001 \\
\hline \multicolumn{3}{|l|}{ Emotional suffering*** } \\
\hline Always (1) & -0.079 & 0.013 \\
\hline Often (2) & -0.065 & -0.009 \\
\hline Sometimes (3) & 0.049 & 0.001 \\
\hline Rarely (4) & 0.094 & -0.004 \\
\hline \multicolumn{3}{|l|}{ Dignity*** } \\
\hline Never (1) & -0.173 & -0.036 \\
\hline Only a little (2) & -0.080 & -0.008 \\
\hline Some (3) & 0.077 & 0.019 \\
\hline Most (4) & 0.176 & 0.025 \\
\hline \multicolumn{3}{|l|}{ Support*** } \\
\hline Never (1) & -0.163 & -0.035 \\
\hline Only a little (2) & -0.058 & -0.017 \\
\hline Some (3) & 0.082 & 0.029 \\
\hline Most (4) & 0.140 & 0.023 \\
\hline \multicolumn{3}{|l|}{ Preparation*** } \\
\hline Not any (1) & -0.146 & -0.017 \\
\hline Only few (2) & -0.020 & -0.010 \\
\hline Some (3) & 0.058 & 0.006 \\
\hline Most (4) & 0.108 & 0.021 \\
\hline \multicolumn{3}{|l|}{ Gender*** } \\
\hline Male & -0.127 & 0.127 \\
\hline Female & 0.109 & -0.109 \\
\hline Diverse & 0.017 & -0.017 \\
\hline
\end{tabular}


Table 4 (continued)

\begin{tabular}{|c|c|c|}
\hline Parameter estimates & Class $1(57.4 \%)$ & Class $2(42.6 \%)$ \\
\hline \multicolumn{3}{|l|}{$\overline{\text { Education } * * *}$} \\
\hline No education & -0.988 & 0.988 \\
\hline Secondary school & 0.006 & -0.006 \\
\hline Intermediate secondary school & 0.210 & -0.210 \\
\hline Technical college & 0.355 & -0.355 \\
\hline A level exam & 0.543 & -0.543 \\
\hline Other education & -0.125 & 0.125 \\
\hline \multicolumn{3}{|l|}{ Employment* } \\
\hline Employed & 0.052 & -0.052 \\
\hline Self-employed & 0.221 & -0.221 \\
\hline Housewife/househusband & 0.199 & -0.199 \\
\hline Not employed & -0.032 & 0.032 \\
\hline Retired & -0.028 & 0.028 \\
\hline Apprenticeship & 0.005 & -0.005 \\
\hline Other employment & -0.416 & 0.416 \\
\hline \multicolumn{3}{|c|}{ Diagnosis of a life limiting illness $* * *$} \\
\hline Yes & -0.338 & 0.338 \\
\hline No & 0.300 & -0.300 \\
\hline No answer & 0.038 & -0.038 \\
\hline \multicolumn{3}{|c|}{ Caring for someone with life limiting illness $* *$} \\
\hline Yes & 0.107 & -0.107 \\
\hline No & -0.104 & 0.104 \\
\hline No answer & -0.004 & 0.004 \\
\hline \multicolumn{3}{|l|}{ Religiosity* } \\
\hline 1 (extremely non-religious) & -0.089 & 0.089 \\
\hline 2 & 0.073 & -0.073 \\
\hline 3 & 0.113 & -0.113 \\
\hline 4 & -0.261 & 0.261 \\
\hline 5 & -0.074 & 0.074 \\
\hline 6 & -0.221 & 0.221 \\
\hline 7 & 0.010 & -0.010 \\
\hline 8 & -0.061 & 0.061 \\
\hline 9 & -0.212 & 0.212 \\
\hline 10 (extremely religious) & -0.355 & 0.355 \\
\hline No answer & 1.051 & -1.051 \\
\hline Class membership parameters & -0.225 & 0.225 \\
\hline \multicolumn{3}{|l|}{ Scaling factors } \\
\hline BWS data*** & -2.582 & \\
\hline BWS-DCE data*** & 1.235 & \\
\hline \multicolumn{3}{|l|}{ Goodness of fit } \\
\hline LL & $-200,256$ & \\
\hline Number of parameters & 89 & \\
\hline AIC & 400,689 & \\
\hline BIC & 401,224 & \\
\hline Number of choice sets & 143,808 & \\
\hline Number of subjects & 2996 & \\
\hline
\end{tabular}

All attributes and class membership covariates are effects coded. Reference levels reported here are calculated as the negative sum of other parameters of the same attribute. Be careful with the coding for 'physical suffering' and 'emotional suffering' (levels are coded reverse)

$B W S$ best-worst-scaling, $D C E$ discrete choice experiment, $L L$ log-likelihood, AIC Akaike information criterion, BIC Bayesian information criterion

$* p \leq 0.05, * * p \leq 0.01, * * * p \leq 0.001$ 
Table 5 Tariff increases and reductions for scale levels of the ICECAP-SCM attributes to calculate tariffs for ICECAP-SCM capability states based on best-worst-scaling, discrete choice experiment and combined data

\begin{tabular}{|c|c|c|c|c|c|c|c|}
\hline $\begin{array}{l}\text { Attribute } \\
\text { level }\end{array}$ & Choice & Love and affection & Physical suffering & Emotional suffering & Dignity & Support & Preparation \\
\hline \multicolumn{8}{|l|}{ BWS data } \\
\hline 1 & 0.008 & -0.004 & -0.003 & 0.019 & -0.040 & -0.007 & 0.027 \\
\hline 2 & 0.030 & 0.043 & 0.025 & 0.014 & 0.010 & 0.045 & 0.087 \\
\hline 3 & 0.098 & 0.108 & 0.081 & 0.071 & 0.085 & 0.115 & 0.120 \\
\hline 4 & 0.143 & 0.164 & 0.141 & 0.101 & 0.142 & 0.160 & 0.149 \\
\hline \multicolumn{8}{|l|}{ DCE data } \\
\hline 1 & -0.002 & -0.020 & 0.015 & 0.041 & -0.069 & 0.005 & 0.029 \\
\hline 2 & 0.072 & 0.061 & 0.053 & 0.068 & 0.049 & 0.063 & 0.072 \\
\hline 3 & 0.104 & 0.115 & 0.112 & 0.098 & 0.129 & 0.046 & 0.091 \\
\hline 4 & 0.139 & 0.157 & 0.132 & 0.106 & 0.204 & 0.141 & 0.121 \\
\hline \multicolumn{8}{|c|}{ Combined data } \\
\hline 1 & 0.019 & 0.016 & -0.024 & 0.013 & -0.052 & -0.024 & 0.052 \\
\hline 2 & 0.042 & 0.052 & -0.009 & -0.006 & 0.017 & 0.039 & 0.111 \\
\hline 3 & 0.139 & 0.065 & 0.042 & 0.051 & 0.111 & 0.147 & 0.161 \\
\hline 4 & 0.169 & 0.167 & 0.081 & 0.064 & 0.158 & 0.164 & 0.198 \\
\hline
\end{tabular}

$B W S$ best-worst-scaling, $D C E$ discrete choice experiment

The tariffs for the capability state " 1112111 " based on BWS and combined data were negative, because respondents rated 'always experiencing emotional suffering' to be less severe than 'often experiencing emotional suffering'. As participants never compared both scale levels directly with each other, is was expected that in German the difference between both scale levels may not be pronounced as in English. Therefore, it was assumed that the tariff of the capability state " 1112111 " is very similar to the tariff of the capability state " 1111111 ", and was thus it replaced by zero

more complex than BWSs by comparing sets of profiles with each other. Therefore, DCEs are able to capture the common influence of attributes. At the same time, DCEs are cognitively more difficult than BWSs and might overburden some participants. Thus, participants may abandon the survey, or answers may depend on the participants' cognitive ability to understand the DCE tasks. Persons with low education or in extraordinary situations (such as the end of life) might be disadvantaged. In contrast to DCEs, BWSs focus on the best and worst statement in one particular capability state. As BWSs are structured more simply, they are much easier to understand and therefore might lead to more valid results.

The experimental design used in this study was based on the UK valuation exercise. This design was more complex compared with most of the published DCE models $[33,33,34]$. It allowed two-way interactions for combined data between different attributes of the ICECAP-SCM. Unfortunately, only solutions of the models including interactions between intercepts of the attributes of the ICECAPSCM and socio-demographic variables were robust. Solutions including interactions between attributes were not robust. The UK study was able to estimate a selected number of interactions for combined data. As the sample size of the UK study ( $n=6020)$ was twice as large as the sample size of the current study ( $n=2996)$, non-robust solutions in the current study may be due to the smaller sample size.
Unfortunately, existing methodological approaches to calculate the required sample size were not transferable to the complex experimental design of the current study. Therefore, robust solutions based on combined data for the current study were derived by analyzing a scale-adjusted conditional logit model with only main effects.

As the statistical analyses based on BWS data was similar for tariffs form German and UK, results are comparable. The tariffs from the UK based on BWS data were normally distributed with mean 0.51, similar to the German tariffs of the current study [19]. Furthermore, the ICC was significant with 0.943 , indicating a strong correlation. Yet, coefficients of different scale levels of attributes varied between the German tariffs and the tariffs from the UK based on BWS data. In particular, tariff increases and reductions for different scale levels of the attributes 'choice', 'emotional suffering', 'dignity', and 'preparation' varied more for German tariffs than UK tariffs. Thus, German respondents compared with UK respondents graded the influence of 'choice', 'emotional suffering', 'dignity', and 'preparation' to be more relevant for capability. On the other hand, tariff increases and reductions for different scale levels of the attributes 'love and affection', 'physical suffering', and 'support' varied less for German tariffs than UK tariffs. Thus, German respondents, when compared with UK respondents, graded the influence of 'love and affection', 'physical suffering', and 'support' to 
be less relevant for capability. Another difference between the analyses of German and UK data was that fewer latent classes were included into the current analyses, compared with the UK evaluation. Models with four latent classes were chosen for BWS and combined data to determine UK tariffs. Thereby, the UK respondents in class 1 graded the influence of 'support' and 'emotional suffering' as less relevant for capability based on BWS data. UK respondents in class 2 considered 'dignity', 'choice' and 'support' as most relevant for capability. For UK respondents in class 3 'love and affection', 'support' and 'dignity' were relevant, whereas UK respondents in class 4 put emphasis on 'support' and 'physical suffering'. Based on combined data, UK respondents in class 1 graded the influenced of 'support' as less relevant. UK respondents in class 2 considered 'love and affection' and 'support' as most relevant for capability. For UK respondents in class 3 'physical suffering' was most relevant, whereas UK respondents in class 4 put emphasis on 'dignity' and 'choice'. German tariffs were based on models with two latent classes. Based on BWS data, German respondents in class 2 graded differences in scale levels of attributes of the ICECAP-SCM higher than German respondents in class 1. Compared with this, German respondents in the DCE graded differences in scale levels of attributes similar in both class.

In conclusion, tariffs for Germany based on BWS, DCE and combined data were similar. However, some differences were observed and have been discussed above. As the analysis of combined data was based on the largest dataset and therefore included the highest amount of information, we would recommend to use tariffs based on combined data in German evaluations. However, as models based on combined data neglected interactions, tariffs based on BWS data may be more comparable between Germany and the UK. This was also suggested for the use of UK tariffs, as former studies for other capability measures (e.g. ICECAP-A [11]) determined tariffs based on BWS data [19].

\section{Implications}

By providing tariffs for the ICECAP-SCM a capability measure is now available to be used as effect measure in health economic analyses in Germany in addition to health-related quality of life. As health-related quality of life measured by the EQ-5D is commonly used to assess health effects in economic evaluations, economic evaluations for end of life interventions should not solely use the ICECAP-SCM. In fact, the use of ICECAP-SCM may compensate for disadvantages of the EQ-5D [7], so that effects of interventions could be captured in a more holistic way. Especially for persons at the end of life the concept of health-related quality of life becomes less relevant, whereas wellbeing becomes more relevant [4-6]. Thus, the capability approach focusing on what persons are able to do and be, depending on what is important to them [8-10], seems to be more suitable to capture effects for interventions at the end of life.

\section{Strength and limitations}

By providing tariffs for the ICECAP-SCM derived from the German general adult population, the ICECAP-SCM will be increasingly practicable for German studies, because tariffs allow to compare the values for capability states at the end of life across medical indications and across countries. Furthermore, the analyses of the current study benefited from a complex experimental design combining DCE and BWS data. Such a complex experimental design allows to capture the best aspects of both approaches: the relative ease of participants to intuitively understand BWS tasks, and the complexity of DCE data, where sets of profiles are compared with each other.

However, as such a complex experimental design has high methodological requirements, the current study has some limitations. First, participants who did not complete the survey or were excluded from the analysis were on average younger and older compared with the German general population. Younger persons $(<35$ years old), who did not complete the survey or were excluded from the analyses, may have found it difficult to put themselves in the position of a person at his or her end of life. For older people ( $>65$ years old) it was expected that these persons were underrepresented in the current study due to the lower affinity with computer technology compared with younger persons in general. Second, the design of the experiments in the current study was based on the design of the UK valuation of the ICECAP-SCM in order to derive comparable results. However, compared with the experimental design of the UK valuation based on combined data, interactions between attributes were not included, thus only main effects in the models with combined data in the current study were included. Models including interactions did not converge and were therefore not included into the current analyses. Even though the experiments have been based on a relatively large sample size of $n=2996$, a larger sample size might result in robust model solutions. Nevertheless, results of the BWS, DCE and combined data were consistent and a larger sample size was not expected to lead to additional information.

\section{Conclusions}

The capability of persons at the end of life can be evaluated using German tariffs of the ICECAP-SCM. The tariffs allow for comparison of capability at the end of life across medical indications and across countries. Thus, the German ICECAP-SCM can be used as effectiveness measure in health economic analyses in addition to health-related quality of life. 


\section{Appendix}

See Fig. 1.

Acknowledgements We would like to thank Joanna Coast and Thomas Grochtdreis for their helpful comments on our manuscript.

Author contributions JD, EH and HHK conceived the study and developed the design. JD war responsible for the analysis and wrote the first draft. All authors contributed to the revision and final approval.

Funding Open Access funding enabled and organized by Projekt DEAL.. This research was funded by the Federal Ministry of Education and Research (Grand number 01GL1725B).

Data availability Data cannot be accessed by anyone who is not part of the research team due to ethical and confidentiality concerns.

Code availability The code can be provided for the reviewing process if needed.

\section{Compliance with ethical standards}

Conflict of interest The authors declare that they have no competing interest.

Open Access This article is licensed under a Creative Commons Attribution 4.0 International License, which permits use, sharing, adaptation, distribution and reproduction in any medium or format, as long as you give appropriate credit to the original author(s) and the source, provide a link to the Creative Commons licence, and indicate if changes were made. The images or other third party material in this article are included in the article's Creative Commons licence, unless indicated otherwise in a credit line to the material. If material is not included in the article's Creative Commons licence and your intended use is not permitted by statutory regulation or exceeds the permitted use, you will need to obtain permission directly from the copyright holder. To view a copy of this licence, visit http://creativecommons.org/licenses/by/4.0/.

\section{References}

1. Calvert, M., Kyte, D., Mercieca-Bebber, R., Slade, A., Chan, A.W., King, M.T., Group, a.t.S.-P: Guidelines for inclusion of patient-reported outcomes in clinical trial protocols: the SPIRITPRO extension guidelines for inclusion of patient-reported outcomes in clinical trial protocols guidelines for inclusion of patientreported outcomes in clinical trial protocols. JAMA 319(5), 483-494 (2018). https://doi.org/10.1001/jama.2017.21903

2. Johnston, B., Flemming, K., Narayanasamy, M.J., Coole, C., Hardy, B.: Patient reported outcome measures for measuring dignity in palliative and end of life care: a scoping review. BMC Health Serv. Res. 17(1), 574 (2017). https://doi.org/10.1186/s1291 3-017-2450-6

3. Mularski, R.A., Dy, S.M., Shugarman, L.R., Wilkinson, A.M., Lynn, J., Shekelle, P.G., Morton, S.C., Sun, V.C., Hughes, R.G., Hilton, L.K., Maglione, M., Rhodes, S.L., Rolon, C., Lorenz, K.A.: A systematic review of measures of end-of-life care and its outcomes. Health Serv. Res. 42(5), 1848-1870 (2007). https://doi. org/10.1111/j.1475-6773.2007.00721.x
4. Patrick, D.L., Engelberg, R.A., Curtis, J.R.: Evaluating the quality of dying and death. J. Pain Symptom Manag. 22(3), 717-726 (2001)

5. Singer, P.A., Martin, D.K., Kelner, M.: Quality end-of-life care: patients' perspectives. Jama 281(2), 163-168 (1999)

6. Steinhauser, K.E., Clipp, E.C., McNeilly, M., Christakis, N.A., McIntyre, L.M., Tulsky, J.A.: In search of a good death: observations of patients, families, and providers. Ann. Intern. Med. 132(10), 825-832 (2000). https://doi.org/10.7326/0003-4819-13210-200005160-00011

7. Davis, M.P., Hui, D.: Quality of life in palliative care. Expert Rev. Qual. Life Cancer Care 2(6), 293-302 (2017). https://doi. org/10.1080/23809000.2017.1400911

8. Nussbaum, M.: Capabilities as fundamental entitlements: sen and social justice. Fem. Econ. 9(2-3), 33-59 (2003). https://doi. org/10.1080/1354570022000077926

9. Sen, A.: The standard of living: lecture II, lives and capabilities. In: Sen, A. (ed.) The standard of living, pp. 20-36. Cambridge University Press, Cambridge (1987)

10. Sen, A.: Capability and well-being. In: Nussbaum, M.C. (ed.) The quality of life. Clarendon Press, Oxford (1993)

11. Al-Janabi, H., Flynn, T.N., Coast, J.: Development of a self-report measure of capability wellbeing for adults: the ICECAP-A. Qual. Life Res. Int. J. Qual. Life Asp. Treat. Care Rehabil. 21(1), 167176 (2012). https://doi.org/10.1007/s11136-011-9927-2

12. Grewal, I., Lewis, J., Flynn, T., Brown, J., Bond, J., Coast, J.: Developing attributes for a generic quality of life measure for older people: preferences or capabilities? Soc. Sci. Med. (1982) 62(8), 1891-1901 (2006). https://doi.org/10.1016/j.socsc imed.2005.08.023

13. Kinghorn, P., Robinson, A., Smith, R.D.: Developing a capability-based questionnaire for assessing well-being in patients with chronic pain. Soc. Indic. Res. 120(3), 897-916 (2015). https://doi. org/10.1007/s11205-014-0625-7

14. Simon, J., Anand, P., Gray, A., Rugkasa, J., Yeeles, K., Burns, T.: Operationalising the capability approach for outcome measurement in mental health research. Soc. Sci. Med. 1982(98), 187-196 (2013). https://doi.org/10.1016/j.socscimed.2013.09.019

15. Davis, J., Liu-Ambrose, T., Richardson, C., Bryan, S.: A comparison of the ICECAP-O and EQ-5D in a falls prevention clinical setting: are they complements or substitutes. Qual. Life Res. Int. J. Qual. Life Asp. Treat. Care Rehabil. (2012). https://doi. org/10.1007/s11136-012-0225-4

16. Keeley, T., Coast, J., Nicholls, E., Foster, N., Jowett, S., AlJanabi, H.: An analysis of the complementarity of ICECAP-A and EQ-5D-3 L in an adult population of patients with knee pain. Health Qual. Life Outcomes (2016). https://doi.org/10.1186/s1295 5-016-0430-x

17. Coast, J.: Strategies for the economic evaluation of end-of-life care: making a case for the capability approach. Expert Rev. Pharmacoecon. Outcomes Res. (2014). https://doi.org/10.1586/14737 167.2014.914436

18. Sutton, E.J., Coast, J.: Development of a supportive care measure for economic evaluation of end-of-life care using qualitative methods. Palliat. Med. 28(2), 151-157 (2014). https://doi. org/10.1177/0269216313489368

19. Huynh, E., Coast, J., Rose, J., Kinghorn, P., Flynn, T.: Values for the ICECAP-Supportive Care Measure (ICECAP-SCM) for use in economic evaluation at end of life. Soc. Sci. Med. 1982(189), 114-128 (2017). https://doi.org/10.1016/j.socscimed.2017.07.012

20. Louviere, J.J., Hensher, D.A., Swait, J.D.: Stated choice methods: analysis and applications. Cambridge University Press, Cambridge (2000)

21. Louviere, J.J., Flynn, T.N., Marley, A.A.J.: Best-worst scaling. Theory, methods and applications. Cambridge University Press, Cambridge (2015) 
22. Coast, J., Flynn, T.N., Natarajan, L., Sproston, K., Lewis, J., Louviere, J.J., Peters, T.J.: Valuing the ICECAP capability index for older people. Soc. Sci. Med. 67(5), 874-882 (2008). https://doi. org/10.1016/j.socscimed.2008.05.015

23. Flynn, T.N., Louviere, J.J., Peters, T.J., Coast, J.: Best-worst scaling: what it can do for health care research and how to do it. J. Health Econ. 26(1), 171-189 (2007). https://doi.org/10.1016/j. jhealeco.2006.04.002

24. Bailey, C., Kinghorn, P., Hewison, A., Radcliffe, C., Flynn, T.N., Huynh, E., Coast, J.: Hospice patients' participation in choice experiments to value supportive care outcomes. BMJ Support. Palliat. Care (2018). https://doi.org/10.1136/bmjspcare-2018001582

25. Krucien, N., Watson, V., Ryan, M.: Is best-worst scaling suitable for health state valuation? A comparison with discrete choice experiments. Health Econ. 26(12), e1-e16 (2017). https://doi. org/10.1002/hec.3459

26. Dorow, M., Stein, J., Liegert, P., Edwards, C., Riedel-Heller, S.: German verison of the ICECAP-SCM. https://www.birmingham .ac.uk/research/activity/mds/projects/HaPS/HE/ICECAP/Evalu ation-of-End-of-Life-Care/ICECAP-SCM.aspx (2019). Accessed 25 Jun 2019

27. Gerlinger, C., Bamber, L., Leverkus, F., Schwenke, C., Haberland, C., Schmidt, G., Endrikat, J.: Comparing the EQ-5D-5L utility index based on value sets of different countries: impact on the interpretation of clinical study results. BMC Res. Notes 12(1), 18 (2019). https://doi.org/10.1186/s13104-019-4067-9
28. Kagawa-Singer, M., Padilla, G.V., Ashing-Giwa, K.: Healthrelated quality of life and culture. Semin. Oncol. Nurs. 26(1), 59-67 (2010). https://doi.org/10.1016/j.soncn.2009.11.008

29. Coast, J., Huynh, E., Kinghorn, P., Flynn, T.: Complex valuation: applying ideas from the complex intervention framework to valuation of a new measure for end-of-life care. PharmacoEconomics (2015). https://doi.org/10.1007/s40273-015-0365-9

30. McFadden, D.: Conditioinallogit analysis of qualitative choice behaviour. In: Zarembka, P. (ed.) Frontiers in econometrics. Academic Press, New York (1974)

31. Flynn, T.N., Huynh, E., Peters, T.J., Alanabi, H., Clemens, S., Moody, A., Coast, J.: Scoring the Icecap-a capability instrument. Estimation of a UK general population tariff. Health Econ 24(3), 258-269 (2015). https://doi.org/10.1002/hec.3014

32. Federal Statistical Offices: Zensus 2011 [Census 2011]. https:// www.zensus2011.de/ (2011). Accessed 11 Jun 2019

33. Clark, M.D., Determann, D., Petrou, S., Moro, D., de BekkerGrob, E.W.: Discrete choice experiments in health economics: a review of the literature. PharmacoEconomics 32(9), 883-902 (2014). https://doi.org/10.1007/s40273-014-0170-x

34. Soekhai, V., de Bekker-Grob, E.W., Ellis, A.R., Vass, C.M.: Discrete choice experiments in health economics: past, present and future. PharmacoEconomics 37(2), 201-226 (2019). https://doi. org/10.1007/s40273-018-0734-2

Publisher's Note Springer Nature remains neutral with regard to jurisdictional claims in published maps and institutional affiliations. 